\title{
De-Noising of ECG Signals by Design of an Optimized Wavelet
}

\author{
Vahid Makhdoomi Kaviri' ${ }^{1}$ Masoud Sabaghi2 ${ }^{*}$, Saeid Marjani ${ }^{3}$ \\ ${ }^{1}$ Department of Electrical Engineering, Islamshar Branch, Islamic Azad University, Tehran, Iran \\ ${ }^{2}$ Laser and Optics Research School, Nuclear Science and Technology Research School, Atomic Energy Organization of Iran, Tehran, Iran \\ ${ }^{3}$ Department of Electrical Engineering, Ferdowsi University of Mashhad, Mashhad, Iran \\ Email: *msabaghi@aeoi.org.ir
}

How to cite this paper: Kaviri, V.M., Sabaghi, M. and Marjani, S. (2016) De-Noising of ECG Signals by Design of an Optimized Wavelet. Circuits and Systems, 7, 3746-3755. http://dx.doi.org/10.4236/cs.2016.711314

Received: April 30, 2016

Accepted: May 25, 2016

Published: September 16, 2016

Copyright $\odot 2016$ by authors and Scientific Research Publishing Inc. This work is licensed under the Creative Commons Attribution International License (CC BY 4.0).

http://creativecommons.org/licenses/by/4.0/

\begin{abstract}
In this paper, a different method for de-noising of ECG signals using wavelets is presented. In this strategy, we will try to design the best wavelet for de-nosing. Genetic algorithm tests wide range of quadrature filter banks and the best of them will be chosen that minimize the Signal-to-Noise Ratio (SNR). Furthermore, the wavelet function and scaling function related to these filters are reported as the best wavelet for de-noising. Simulation results for de-noising of a noisy ECG signal show that using obtained wavelet by proposed method improves the SNR of about $2.5 \mathrm{~dB}$.
\end{abstract}

\section{Keywords}

Wavelets, De-Noising, Genetic Algorithm, ECG Signals

\section{(c) (†) Open Access}

\section{Introduction}

There are many methods to de-noise a signal. De-noising is so important in signal processing, particularly for biomedical signals. Discrete Wavelet Transform (DWT) is currently used in a wide variety of signal processing applications, such as audio and video compression, removal of noise in audio, and the simulation of wireless antenna distribution. The wavelet transforms have good properties such as the time-frequency localization, energy compaction and sub-band coding. Wavelet transform is one of the most powerful mathematical tools for digital signal processing [1], such as compression and de-noising.

Genetic algorithm (GA) is an optimization technique based on the "survival of the fittest" [2]. In this method, a wide range of inputs are checked and the best one of them is chosen. Genetic algorithm calculates the fitness of the solution according to fitness 
function by search approximately all of the possible inputs. Discrete wavelet transform is implemented by digital FIR filters.

When the voltage of the ECG waveform is at least larger than $75 \%$ of the peak value of the ECG in the comparator stage (digital-comparator) [3]-[5] of the peak detector, it allows oscillation generator to be fed into speaker for beeping. Digitizing ECG signals carry out using successive approximation ADC control system [6] [7] or delta-sigma analog to digital converter (decimation filter) [8]-[12]. In order to generate an error signal, a subtractor configures to subtract the filtered ECG signal that is generated by the adder [13] from the ECG signal input to the inputter. After proper amplification and filtration of the ECG signal, it is given to a voltage controlled oscillator (VCO) [14].

The ECG signal is a reference signal for pulse wave delay using photoplethysmographic signal and Laser-Doppler (LD) measurements. The basis of the registration is the selfmixing in the diode lasers cavities [15]-[25]. Also, the ECG signals are going to be transmitted into laser beam such as VCSELs [26]-[38] to stablish communication between the ECG and the medical center.

In this paper, the best coefficients of filters will be obtained using genetic algorithm. GA searches several coefficients and thresholds to reach the best output SNR. The paper is organized as follows: in Section 2, de-noising using wavelet implementation is presented. In Section 3, genetic algorithm is introduced and then the proposed method is developed. Simulation results are presented in Section 4. Finally conclusion is given in Section 5.

\section{Wavelet Based De-Noising}

The process of removing the noise, $e[n]$, from a signal, $\hat{x}[n]=x[n]+e[n]$ is called "de-nosing". For example, $e[n]$ may be a Gaussian white noise process, which is statistically independent of $x[n]$. A method for de-noising is the applying a nonlinear operation to a representation of $\hat{x}[n]$, like fourier transform or wavelet transform. In this work, wavelet transform is chosen. The de-noising procedure is as follows: First, the signal $\hat{x}[n]$ is decomposed using a filter bank, thus performing discrete wavelet transform. Then, the wavelet coefficients are manipulated in order to remove the noise component. Two approaches known as hard and soft thresholding have been proposed for this purpose. In hard thresholding, coefficients that their absolute values are smaller than a specific threshold, are replaced with zero. The idea of thresholding is that $x[n]$ can be represented via a number of wavelet coefficients, while the noise has wideband characteristics and spreads out on all coefficients. Thus, it provided the threshold $\varepsilon$ is chosen appropriately, the signal constructed from the manipulated wavelet coefficients will contain much less noise than $\hat{x}[n]$ does [39] [40]. On the other hand, the waveform of used wavelet is so important for de-noising (or compression). In [41] [42], several de-noising methods using wavelets are compared.

\section{Wavelet Design Using Genetic Algorithm}

In this section after a brief summary of genetic algorithm, the concept of multi-resolution 
analysis and the efficient realization of the discrete wavelet transform based on multi-rate filter banks are presented. Then, the proposed method is discussed.

\subsection{Genetic Algorithm (GA)}

Genetic Algorithm is used to introduce computer-based problems solving systems, which use computational models of evolutionary processes. Different algorithms have been proposed in literature, such as: GAs, evolutionary programming, evolution strategies, classifier systems, and genetic programming. Via processes of selection, mutation and reproduction, these algorithms present a common conceptual base. The genetic algorithms are based on reproduction, fitness, crossover and mutation. The standard procedure of genetic algorithms is as follow:

1) Candidate solutions to a problem have been started with a randomly generated population of $n 1$-bit strings.

2) Fitness function $f(x)$ of each string in the population is calculated.

3) Until $n$ new strings have been created, the following steps have to repeat:

a) From the current population, a pair of parent strings is selected where probability of selection being an increasing function of fitness.

b) In order to form two new strings, cross over the pair at a random point with the crossover probability.

c) With the mutation probability, the two new strings obtained from previous step mutate at each locus. Then they place the resulting strings in the new population.

4) The current population has to replace with the new population.

5) Go to step 2.

\subsection{Multi-Resolution Analysis for Design of an Appropriate Wavelet for De-Noising}

The main concept of wavelet transform based on multi-resolution analysis are presented in this section. This framework has been developed by Meyer, Mallat and Daubechies mainly, for the orthonormal cases [43] [44]. Design procedure is generally performed by designing a Quadrature Mirror filter Bank (QMFB) with Perfect Reconstruction (PR) conditions [40]. Figure 1 shows the analysis and synthesis filters.

If the output signal be a delayed version of the input signal, perfect reconstruction is obtained. PR conditions for the filter bank that is shown in Figure 1 are:

$$
\begin{aligned}
& H_{1}(-z) F_{1}(z)+H_{2}(-z) F_{2}(z)=0 \\
& H_{1}(z) F_{1}(z)+H_{2}(z) F_{2}(z)=2 z^{-d}
\end{aligned}
$$

Condition (1) says that the output signal contains no aliasing, but amplitude distortions may be occurred. If both (1) and (2) are satisfied, the amplitude distortions are also vanishing. There are many proper filter's coefficients to satisfy in (1), but condition (2) is only complied approximately. Proposed method is based on using Genetic Algorithm (GA) to reach the best filter coefficients. In other words, best wavelet function that results minimum SNR after de-noising. So, some parameters are arbitrary in design 


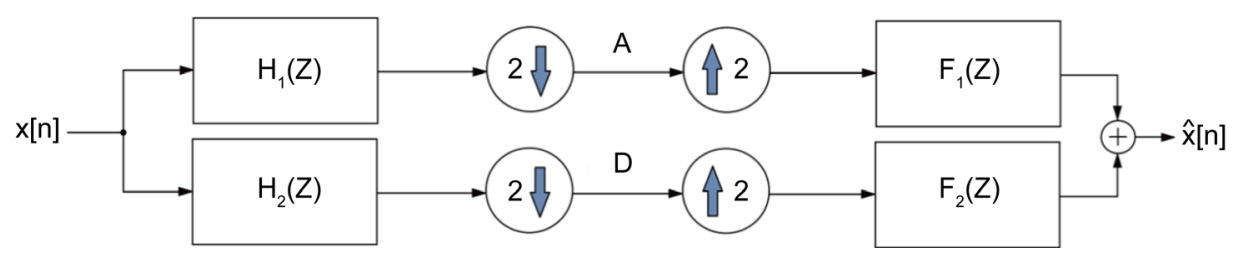

Figure 1. Quadrature mirror filter bank (QMFB).

procedure. A proper set of conditions used vastly in wavelet design are:

$$
\begin{aligned}
& H_{2}(z)=-z^{-l} H_{1}\left(-z^{-1}\right) \\
& F_{1}(z)=H_{2}(-z) \\
& F_{2}(z)=-H_{1}(-z)
\end{aligned}
$$

Note that the $H_{1}(z)$ is analysis low-pass filter. These conditions meet the Equation (1). Therefore, it is enough to find the filters that can satisfy Equation (2) exactly, or approximately. When the number of wavelet levels is not exceeded from 3 or 4 , a nearPR filter bank is sufficient and gives good results. In this work, we use 3-level decomposition and reconstruction.

One chooses the coefficients of a PR two-channel filter bank in such a way that the wavelets and scaling functions associated with these filters have the desired properties. For constructing wavelets, we use two equations called two-scale relations [40]:

$$
\begin{aligned}
& \phi(t)=\sum_{n} f_{1}[n] \sqrt{2} \phi(2 t-n) \\
& \psi(t)=\sum_{n} f_{2}[n] \sqrt{2} \phi(2 t-n)
\end{aligned}
$$

$\phi(t)$ and $\psi(t)$ are called "scaling function" and "waveletfunction", respectively. The starting point to constructing scaling functions is the first part of the two-scale relation. In this manner, first we must select coefficients of reconstruction low pass filter, $f_{1}[n]$, appropriately. To construct biorthogonal and orthonormal scaling functions and wavelets, the coefficients of PR two-channel filter banks are required. Since the scaling function, $\phi(t)$, is supposed to be a low-pass impulse response, generally, it introduces the normalization [40]:

$$
\Phi(0)=\int_{-\infty}^{+\infty} \phi(t) \mathrm{d} t=1
$$

where, $\Phi(\omega)$ is fourier transform of scaling function. By integrating from $\phi(t)$ and $\psi(t)$ in Equations (6) and (7), and this fact that $\int_{-\infty}^{+\infty} \psi(t)=0$, we will obtain:

$$
\begin{aligned}
& \sum_{n} f_{1}[n]=\sqrt{2} \\
& \sum_{n} f_{2}[n]=0
\end{aligned}
$$

If $F_{1}(-1)=0$, a low-pass behavior for $F_{1}(z)$ is achieved. Therefore:

$$
\sum_{n}(-1)^{n} f_{1}[n]=0
$$

From (3), (4), (5), $f_{2}[n]=-(-1)^{n} f_{1}[n]$. Then if $F_{1}(-1)=0$, Equation (10) will be 
satisfied. A simple way to have zeros in $z=-1$ is to use a Type-II symmetric linear phase FIR filter for $f_{1}[n]$. An suitable set of coefficients can be realized when condition (2) is satisfied. $\phi(t)$ and $\psi(t)$ should possibly have been several continuous derivatives. A test that can check the regularity of the product is introduced by Daubechies. Assuming that $F_{1}(z)$ has $N$ zeros in $z=-1, \quad F_{1}(z)$ can be written as [40]:

$$
F_{1}(z)=\sqrt{2} R(z) \frac{\left(1+z^{-1}\right)^{N}}{2^{N}}
$$

In order to achieving smooth wavelets with continuous derivatives, three zeros in $Z=$ -1 are considered. Then, Equation (12) can be written as:

$$
F_{1}(z)=\left(\frac{\sqrt{2}}{8}\right) R(z)\left(1+3 z^{-1}+3 z^{-2}+z^{-3}\right)
$$

As seen from Equation (9), $R(1)=1$. Therefore, $R(z)$ can be considered as a polynomial of $z^{-1}$, with $\sum_{n} r[n]=1$. If $f_{1}[n]$ is a low-pass filter with 8 coefficients and three zeros in $z=-1, R(z)$ is a FIR system with five coefficient where its summation is equal to 1 .

\subsection{GA Strategy for Design Optimum Coefficients to Minimize the SNR}

In this work, a 3-level wavelet decomposition is implemented. The FIR filters have 8coefficients. Then, thresholding and reconstruction are performed on sub-bands to obtain the de-noised signal. The strategy is as follows: Fur coefficients of $r[n]$ is arbitrary chosen as first population for genetic algorithm. The 8 coefficients that constructing low-pass filter, $F_{1}(z)$, are made according to realization of the discrete wavelet transform based on multi-rate filter banks. Figure 2 shows a 3-level thresholding and reconstruction to attain the de-noised signal. In GA technique, thresholds also considered for all sub-bands to finding the best filter coefficients (used wavelet) and thresholds which minimize the SNR.

\section{Simulation Results}

We consider a smooth and noiseless ECG signal as a reference to calculate SNR. A Gaussian White Noise (GWN) is added to this pure signal to make a noisy ECG with a defined SNR. Then, the proposed algorithm is applied on this noisy signal and results are compared with other wavelets de-noising from a SNR point of view. Obtained filter coefficients define a wavelet function and a scaling function, which also will report. Simulation results are presented in Table 1: the wavelet that introduced by the proposed method improve the output SNR about $2.5 \mathrm{~dB}$ more than other wavelets implemented by means of filters which have 8 coefficients.

Figure 3 shows the noisy input signal and de-noised output signal using the proposed method for two situations. For an input SNR of $36.7 \mathrm{~dB}$, the output SNR is 48.7 $\mathrm{dB}$ and for an input SNR of $45.2 \mathrm{~dB}$, the output SNR is $59.2 \mathrm{~dB}$. It's clear that if we use the filters that have more coefficients, the results will be improved. Finally, Figure 4 


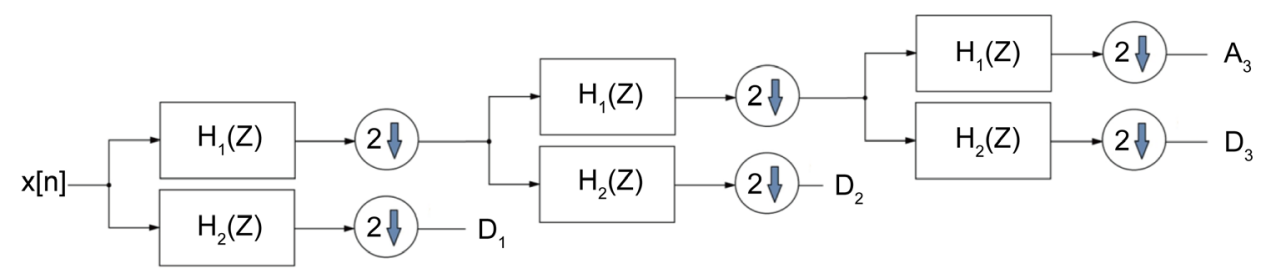

(a)

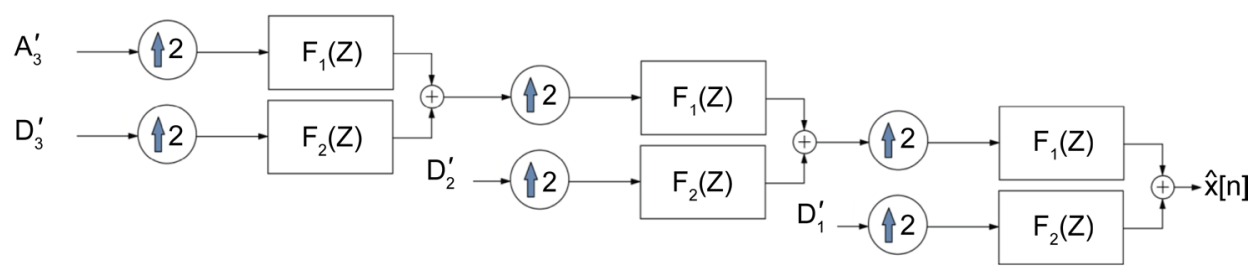

(b)

Figure 2. 3-level wavelet implementation. (a) Decomposition; (b) Reconstruction.

(a)
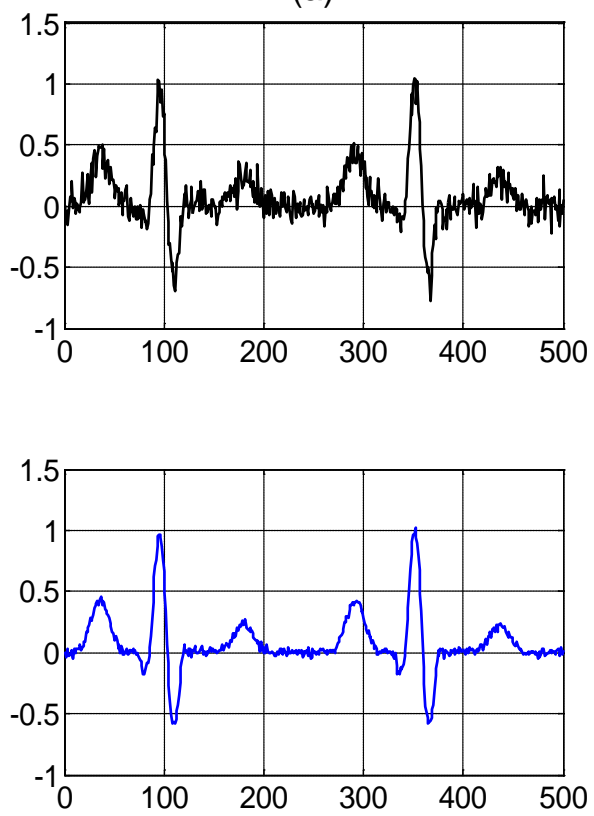

(b)
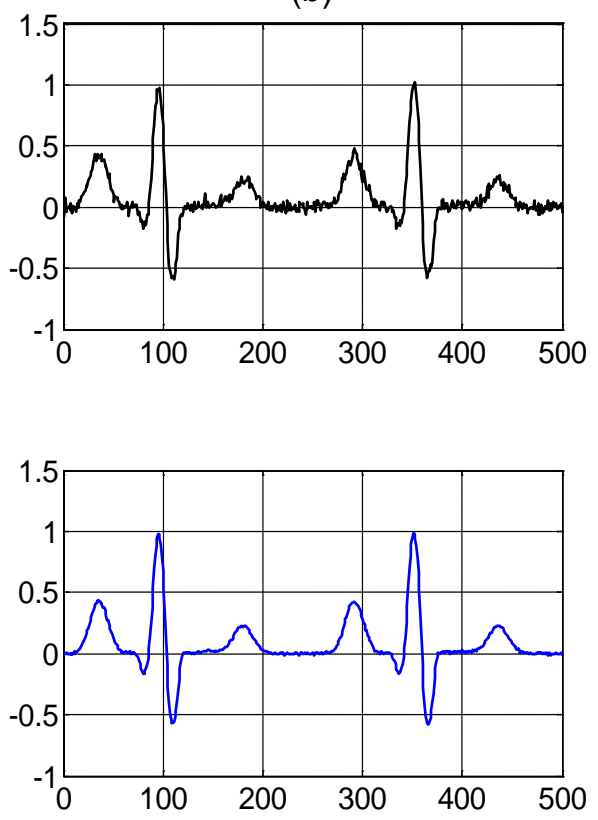

Figure 3. (a) The noisy and de-noised signal using proposed method (input SNR $=36.7 \mathrm{~dB}$ and output $\mathrm{SNR}=48.7 \mathrm{~dB}$ ) and (b) noisy and de-noised signal using proposed method (input $\mathrm{SNR}=$ $45.2 \mathrm{~dB}$ and output $\mathrm{SNR}=59.2 \mathrm{~dB}$ ).

Table 1. Output and input SNR for different wavelets.

\begin{tabular}{cccc}
\hline Wavelet name & Input SNR (dB) & Output SNR (dB) & Improve (dB) \\
\hline Bior3.3 & 36.7 & 46.57 & 9.87 \\
Db. 4 & 36.7 & 46.19 & 9.49 \\
Sym. 4 & 36.7 & 46.26 & 9.56 \\
Proposed method & 36.7 & 48.7 & 12 \\
\hline
\end{tabular}



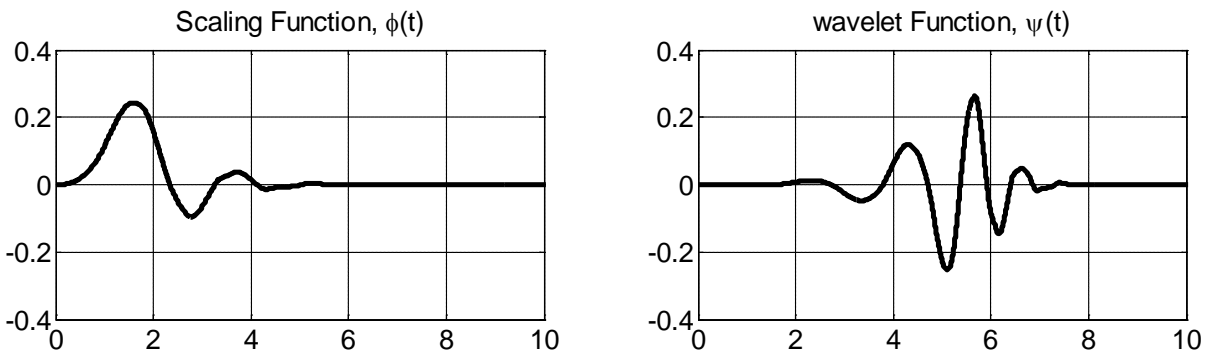

Figure 4. Optimized wavelet function and scaling function.

shows the wavelet function and scaling function constructed from 8-coefficcient filters found by Genetic Algorithm, respectively.

\section{Conclusion}

A new algorithm for de-noising of ECG signals is presented. The method is based on making a specific wavelet function for minimizing the SNR. By using genetic algorithms, the coefficients of wavelet filter bank alter smoothly until the best SNR for output signal achieved. We used this method and de-noised a noisy ECG signal with 3-level wavelet structure that used 8-coefficient-filters and the results were improved in comparison by typical wavelets such as Daubechies 4, Symlet4 and Bior 3.3.

\section{References}

[1] Miao, S.G., Yen, H.L. and Lin, C.H. (2002) Wavelet-Based ECG Compression Using Dynamic Vector Quantization with Tree Code Vectors in Single Code Book. IEEE Transactions on Biomedical Engineering, 49, 671-680.

http://dx.doi.org/10.1109/TBME.2002.1010850

[2] Jones, E., Runkle, P., Dasgupta, N., Couchman, L. and Carin, L. (2001) Genetic Algorithm Wavelet Design for Signal Classification. IEEE Transactions on Pattern Analysis and Machine Intelligence, 23, 890-895. http://dx.doi.org/10.1109/34.946991

[3] Dashtbayazi, M., Sabaghi, M. and Marjani, S. (2015) Dynamic Comparator with Using Negative Resistance and CMOS Input Pair Strategies in FS $=4 \mathrm{MHz}-10 \mathrm{GHz}$. Journal of Electrical and Electronic Engineering, 3, 93-96. http://dx.doi.org/10.11648/j.jeee.20150304.15

[4] Dashtbayazi, M., Marjani, S. and Sabaghi, M. (2015) A 4 MHz - 10 GHz, 10-ps/dec Dynamic Comparator Using Negative Resistance Combined with CMOS Input Pair. The Progress in Electromagnetics Research Symposium (PIERS), Prague, 831-834.

[5] Sabaghi, M., Majdabadi, A., Dashtbayazi, M. and Marjani, S. (2015) A 4 MHz - $10 \mathrm{GHz}$, Dynamic Comparator Using Negative Resistance Combined with CMOS Input Pair Strategy in Dynamic Pre-Amplifier. The Iranian Conference on Optics and Laser Engineering (ICOLE), Isfahan, 136-139.

[6] Dashtbayazi, M., Sabaghi, M. and Marjani, S. (2015) An Optimized DAC Timing Strategy in SAR ADC with Considering the Overshoot Effect. Journal of Electrical and Electronic Engineering, 3, 19-24. http://dx.doi.org/10.11648/j.jeee.20150302.12

[7] Sabaghi, M., Dashtbayazi, M. and Marjani, S. (2016) Dynamic Hysteresis Band Fixed Frequency Current Control. World Applied Programming, 6, 1-4.

http://dx.doi.org/10.21828/WAP-06-01-001 
[8] Dashtbayazi, M., Sabaghi, M., Rezaei, M. and Marjani, S. (2014) New Delta Sigma Modulator Structure Using Second Order Filter in One Stage Technique. Journal of Electrical and Electronic Engineering, 2, 82-88. http://dx.doi.org/10.11648/j.jeee.20140206.11

[9] Sabaghi, M., Dashtbayazi, M. and Marjani, S. (2015) An Optimized Opamp-Sharing in 2nd Order $\Delta \Sigma$ Modulator Based on Changing the Stages Output Capacitance Timing Strategy. The Progress in Electromagnetics Research Symposium (PIERS), Prague, 827-830.

[10] Dashtbayazi, M., Majdabadi, A., Sabaghi, M. and Marjani, S. (2015) An Optimized OpampSharing Technique in 2nd Order Delta-Sigma Modulator Based on Changing the Stages Output Capacitance Timing Strategy. The Iranian Conference on Optics and Laser Engineering (ICOLE), Isfahan, 129-131.

[11] Dashtbayazi, M., Marjani, S., Sabaghi, M. and Majdabadi, A. (2016) Changing the Clock Pulse-Width Dedicated to Stages Strategy for Opamp-Sharing Technique in 2nd Order High-Pass Delta-Sigma Modulators. The International Conference on Electrical, Computer, Mechanical and Mechatronics Engineering (ICE), Dubai, 1-3.

[12] Marjani, S., Sabaghi, M., Majdabadi, A. and Dashtbayazi, M. (2016) An Ultra-Low-Power $\Delta \Sigma$ Modulator Using ZCD-Sharing and Changing Stages Output Capacitance Timing Techniques. The 4th International Conference on Science and Engineering (ICES2016), Rome, $1-5$.

[13] Sabaghi, M., Marjani, S. and Majdabadi, A. (2016) The Design of Ultra-Low Power Adder Cell in 90 and $180 \mathrm{~nm}$ CMOS Technology. Circuits and Systems, 7, 58-67.

[14] Sabaghi, M., Marjani, S. and Majdabadi, A. (2016) A Low Phase Noise, Low Power and Wide Tuning Range VCO with Filtering Technique in ISM Band. Circuits and Systems, 7, 51-57. http://dx.doi.org/10.4236/cs.2016.72006

[15] Marjani, A., Marjani, S. and Shirazian, S. (2011) Numerical Simulation of Silicon Carbide Polymers (6H-SiC \& $3 \mathrm{C}-\mathrm{SiC})$ as the Active Area for $0.83 \mu \mathrm{m}$ Wavelength Semiconductor Laser. The 14th Iranian Physical Chemistry Conference, University of Tehran, Kish, 876878.

[16] Marjani, S., Faez, R. and Marjani, H. (2011) Analysis and Design of Semiconductor Laser with Silicon Carbide Polymers (6H-SiC and 3C-SiC). Australian Journal of Basic and Applied Sciences, 5, 1060-1063.

[17] Marjani, S. and Marjani, H. (2012) Self-Heating Effects in a Silicon Carbide Polymers (6H-SiC and 3C-SiC) Semiconductor Laser. Asian Journal of Chemistry, 24, 3145-3147.

[18] Marjani, S. and Marjani, H. (2012) Effects of Lattice Temperature on the Various Elements of Heat Sources in Silicon Carbide Polymers (6H-SiC and 3C-SiC) Semiconductor Laser. Asian Journal of Chemistry, 24, 3123-3125.

[19] Marjani, S., Faez, R. and Marjani, H. (2012) Analysis of the Various Elements of Heat Sources in Silicon Carbide Polymers (6H-SiC and 3C-SiC) Semiconductor Laser. Asian Journal of Chemistry, 24, 2333-2335.

[20] Marjani, S., Faez, R. and Marjani, H. (2012) Design and Modeling of a Semiconductor Laser by Employing Silicon Carbide Polymers (6H-SiC, $3 \mathrm{C}-\mathrm{SiC}$ and $4 \mathrm{H}-\mathrm{SiC})$. Asian Journal of Chemistry, 24, 2177-2179.

[21] Marjani, S., Faez, R. and Hosseini, S.E. (2013) Analysis of Lattice Temperature Effects on a GaInP/6H-SiC Strained Quantum-Well Lasers. Asian Journal of Chemistry, 25, 4715-4717.

[22] Marjani, S., Faez, R. and Marjani, H. (2013) Silicon Carbide Polymers (6H-SiC, 3C-SiC and $4 \mathrm{H}-\mathrm{SiC}$ ) Semiconductor Laser: Influence of Self-Heating. The 3rd Iranian Conference on Optics and Laser Engineering (ICOLE), Malek Ashtar University of Technology, Isfahan, 8-10 October 2013, 1069-1072. 
[23] Rafighi, F., Behrouzinia, S., Khorasani, K., Sabaghi, M. and Marjani, S. (2016) The Electrical Parameters Modeling and Experimentation of Copper Vapor Laser. Circuits and Systems, 7, 23-28. http://dx.doi.org/10.4236/cs.2016.71003

[24] Behrouzinia, S., Khorasani, K., Marjani, S., Sabaghi, M., Aeinehvand, M.E. and Mohammadpour, S. (2016) Experimental Study of Buffer Gas Flow Rate Effect on Output Power of a Copper Vapor Laser. Optics and Photonics Journal, 6, 24-28.

http://dx.doi.org/10.4236/opj.2016.62004

[25] Mirzaei, M., Behrouzinia, S., Sabaghi, M., Marjani, S., Khorasani, K. and Sajad, B. (2016) Experimental Optimization of the Output Power of a Copper Vapor Laser Using Air as a Buffer Gas. Optics and Photonics Journal, 6, 53-59. http://dx.doi.org/10.4236/opj.2016.64008

[26] Faez, R., Marjani, A. and Marjani, S. (2011) Design and Simulation of a High Power Single Mode 1550nm InGaAsP VCSELs. IEICE Electronics Express, 8, 1096-1101. http://dx.doi.org/10.1587/elex.8.1096

[27] Marjani, S., Faez, R. and Marjani, H. (2011) An Impact of the Hole Etching Depth within a Photonic Crystal VCSEL on Its Heat Sources. Australian Journal of Basic and Applied Sciences, 5, 766-770.

[28] Marjani, S., Faez, R. and Marjan, A. (2011) Design and Modeling of a High Single Mode Power Long Wavelength InGaAsP Photonic Crystal VCSEL. Australian Journal of Basic and Applied Sciences, 5, 1064-1069.

[29] Marjani, S., Rahnama, M. and Marjani, H. (2011) Numerical Optimization of Single-Mode InGaAsP Vertical-Cavity Surface-Emitting Lasers. Australian Journal of Basic and Applied Sciences, 5, 1207-1211.

[30] Marjani, S. and Marjani, H. (2011) Effects of Lattice Temperature on the Various Elements of Heat Sources in a Long Wavelength InGaAsP Photonic Crystal VCSEL. Australian Journal of Basic and Applied Sciences, 5, 1257-1261.

[31] Marjani, S. and Marjani, H. (2011) Analysis of Lattice Temperature Effects on a Long Wavelength InGaAsP Photonic Crystal VCSEL. Australian Journal of Basic and Applied Sciences, 5, 1374-1378.

[32] Marjani, S. and Marjani, H. (2012) Optimization of a Long Wavelength Vertical-Cavity Surface-Emitting Lasers by Employing Photonic Crystal. Asian Journal of Chemistry, 24, 3174-3176.

[33] Marjani, S. and Marjani, H. (2012) Effects of Hole Etching Depth in a Long Wavelength InGaAsP Photonic Crystal Vertical Cavity Surface Emitting Laser. Asian Journal of Chemistry, 24, 3194-3196.

[34] Marjani, S. (2013) Various Elements of Heat Sources within an Optimized Photonic Crystal Vertical Cavity Surface Emitting Laser: Influence of Hole Etching Depth. Asian Journal of Chemistry, 25, 4153-4156.

[35] Marjani, S. (2013) Optimization of an InGaAsP Vertical-Cavity Surface-Emitting Diode Lasers for High-Power Single-Mode Operation in $1550 \mathrm{~nm}$ Optical-Fibre Communication Systems. Asian Journal of Chemistry, 25, 4150-4152.

[36] Marjani, S., Faez, R. and Hosseini, S.E. (2013) Threshold Characteristics Analysis of InP-Based PhC VCSEL with Buried Tunnel Junction. The 21 st Iranian Conference on Electrical Engineering (ICEE), Ferdowsi University of Mashhad, Mashhad, 4-16 May 2013, 1-4. http://dx.doi.org/10.1109/iraniancee.2013.6599783

[37] Majdabadi, A., Marjani, S. and Sabaghi, M. (2014) Threshold Characteristics Enhancement of a Single Mode $1.55 \mu \mathrm{m}$ InGaAsP Photonic Crystal VCSEL for Optical Communication 
Systems. Optics and Photonics Journal, 4, 296-303.

http://dx.doi.org/10.4236/opj.2014.410029

[38] Naeemi, M.A., Marjani, S. and Peiravi, A. (2014) Time to Failure Analysis of Single Mode Long-Wavelength InGaAsP Vertical-Cavity Surface-Emitting Lasers. The 22st Iranian Conference on Electrical Engineering (ICEE), Shahid Beheshti University, Tehran, 20-22 May 2014, 43-47. http://dx.doi.org/10.1109/IranianCEE.2014.6999500

[39] Donoho, D.L. and Johnstone, I.M. (1994) Ideal Spatial Adaptation via Waveletshrinkage. Biometrika, 81, 425-455. http://dx.doi.org/10.1093/biomet/81.3.425

[40] Mertins, A. (2003) Signal Analysis: Wavelet, Filter Banks, Time-Frequency Transform and Applications. John Wiley \& Sons Inc., Hoboken.

[41] Garg, G., Singh, V., Gupta, J.R.P. and Mittal, A.P. (2010) Optimal Algorithm for ECG denoising Using Discrete Wavelet Transforms. The IEEE International Conference on Computational Intelligence and Computing Research (ICCIC), Coimbatore, 28-29 of December 2010, 1-4.

[42] Jaffery, Z.A. and Afroz, A.K. (2010) Performance Comparison of Wavelet Threshold Estimators for ECG Signal Denoising. The IEEE International Conference on Advances in Recent Technologies in Communication and Computing (ARTCom), Kottayam, 16-17 October 2010, 248-251.

[43] Daubechies, I. (1998) Orthonormal Bases of Compactly Supported Wavelets. Communications on Pure and Applied Mathematics, 41, 909-996.

http://dx.doi.org/10.1002/cpa.3160410705

[44] Mallat, S. (1989) A Theory for Multiresolution Signal Decomposition: The Wavelet Representation. IEEE Transactions on Pattern Analysis and Machine Intelligence, 11, 674-693. http://dx.doi.org/10.1109/34.192463

Submit or recommend next manuscript to SCIRP and we will provide best service for you:

Accepting pre-submission inquiries through Email, Facebook, LinkedIn, Twitter, etc. A wide selection of journals (inclusive of 9 subjects, more than 200 journals)

Providing 24-hour high-quality service

User-friendly online submission system

Fair and swift peer-review system

Efficient typesetting and proofreading procedure

Display of the result of downloads and visits, as well as the number of cited articles

Maximum dissemination of your research work

Submit your manuscript at: http://papersubmission.scirp.org/ 